\title{
Relativistic approach to electromagnetic imaging
}

\author{
Neil V. Budko \\ Laboratory of Electromagnetic Research, Faculty of Electrical Engineering, \\ Mathematics and Computer Science, Delft University of Technology, Mekelweg 4, \\ 2628 CD Delft, The Netherlands
}

\begin{abstract}
A novel imaging principle based on the interaction of electromagnetic waves with a beam of relativistic electrons is proposed. Wave-particle interaction is assumed to take place in a small spatial domain, so that each electron is only briefly accelerated by the incident field. In the one-dimensional case the spatial distribution of the source density can be directly observed in the temporal spectrum of the scattered field. Whereas, in the two-dimensional case the relation between the source and the spectrum is shown to be approximately the Radon transform.
\end{abstract}

Key words: electromagnetism, imaging, relativistic electrons, Radon transform, Doppler transform

PACS: 03.50.De, 42.30.Va, 42.30.Wb, 02.30.Uu, 02.30.Zz, 41.60.-m

\section{Introduction}

Telescopes, antenna arrays, radars, and imaging interferometers share a common resolution criterion: the spatial extent of their aperture. Higher resolution means a larger lens, a larger reflector, or a longer interferometer base. At the present stage any significant improvement of the resolution is associated with extreme technical difficulties and costs, and for some applications we are approaching a limit in this respect. Hence, it makes sense to consider alternative imaging principles, especially ones where the attainable resolution would not be entirely controlled by the spatial extent of some 'aperture'.

Email address: n.budko@ewi.tudelft.nl (Neil V. Budko). 
In a variety of cases both the observer and the observed object are in relative motion with respect to each other. A well-known phenomenon related to this motion is the Doppler effect. It is widely exploited in radar [1], and acoustical imaging of blood vessels [2], [3], and is sometimes used to improve the resolution in astronomy [4]. Not to mention the famous red shift of stars and galaxies around us, which is the main source of cosmological information. However, in all of these imaging techniques the observer is actually considered to be at rest. Often, this is just a question of reference frame, and we simply find it convenient to relate ourselves with the one at rest. If for some reason the motion of the observer cannot be neglected in this way, then our natural intention is to compensate for it [5], [6].

In this paper we investigate the possibility of exploiting the observer's motion in electromagnetic imaging, rather than neglecting or correcting for it. Apparently, for this purpose we need an observer that moves with a relativistic velocity. This, however, presents not a problem, since an electron, which is easily accelerated to such velocities, is also the most natural "observer" for the electromagnetic field. An obvious, but not at all unique way to organize an imaging system of this kind is to let a beam of relativistic electrons interact with the fields radiated by some remote spatially inhomogeneous source distribution, say a group of stars. Contrary to standard (stationary) imaging instruments, here it is the observer's velocity, not the spatial extent of the aperture, that determines the attainable resolution.

One of the problems in [7] (p. 249), where a plane monochromatic wave interacts with a single relativistic electron, contains a hint, which has inspired the present paper. In [7] it is claimed that upon scattering another plane wave can be observed in the far-field, which has a different frequency with respect to the incident one, depending on the angles of incidence and observation. Here we simulate an imaging setup and, therefore, consider incident fields due to a remote but spatially extended source. These fields interact with a short segment of a relativistic current. The short-segment assumption allows to circumvent the difficult problem of charge dynamics. Straightforward calculations presented below show that the relation between the spatial density distribution of the source and the (temporal) spectrum of the secondary field radiated by the electrons is approximately a Radon transform. This transform is well known in imaging theory and is amenable to invertion [8]. Mathematicaly, the particular form of the Radon transform obtained here is very similar to the Doppler and Doppler-Radon transforms, which are extensively studied in the (non-relativistic) acoustical imaging of fluid flows [2], [3].

The author does not claim to have covered all physical and mathematical aspects of this interesting problem. In fact, to arrive at the Radon transform in its simplest form we make several approximations, the significance of which must be further investigated both theoretically and experimentally. 


\section{Theory}

Let there be a remote spatially inhomogeneous and time varying source of the electromagnetic field. We consider the scattering of this field by a segment of relativistic electric current, e.g. a beam of electrons moving with a relativistic velocity in a particle accelerator or even a simple cathode ray tube. Neglecting the mutual interaction between the electrons, quantum effects, as well as the radiation reaction force, we would like to calculate the secondary electromagnetic field, scattered by this relativistic current into the far-field zone.

Let $\mathbf{x}_{\mathrm{e}}(t)$ denote the location of a single moving electron and let $D_{\mathrm{s}}$ be a bounded region of space occupied by an external source of the electromagnetic field (see Fig. 1). Presume that $D_{\mathrm{s}}$ is far enough both from the origin of the coordinate system and location $\mathbf{x}_{\mathrm{e}}(t)$, so that the distance factor can be approximated by

$$
\left|\mathbf{x}_{\mathrm{s}}-\mathbf{x}_{\mathrm{e}}\left(t_{\mathrm{e}}\right)\right| \approx\left|\mathbf{x}_{\mathrm{s}}\right|-\mathbf{n}_{\mathrm{s}} \cdot \mathbf{x}_{\mathrm{e}}\left(t_{\mathrm{e}}\right), \quad \text { for } \quad \mathbf{x}_{\mathrm{s}} \in D_{\mathrm{s}}
$$

where $\mathbf{n}_{\mathrm{s}}=\mathbf{x}_{\mathrm{s}} /\left|\mathbf{x}_{\mathrm{s}}\right|$. In Gaussian units the incident electromagnetic field at the location of an electron is given by

$$
\begin{aligned}
& \mathbf{E}^{\text {in }}\left(\mathbf{x}_{\mathrm{e}}, t_{\mathrm{e}}\right)=\frac{1}{c^{2}} \int_{\mathbf{x}_{\mathrm{s}} \in D_{\mathrm{s}}} \frac{\mathbf{n}_{\mathrm{s}} \times\left(\mathbf{n}_{\mathrm{s}} \times \partial_{t_{\mathrm{s}}} \mathbf{J}\left(\mathbf{x}_{\mathrm{s}}, t_{\mathrm{s}}\right)\right)}{\left|\mathbf{x}_{\mathrm{s}}\right|} \mathrm{d} V_{\mathrm{s}}, \\
& \mathbf{H}^{\mathrm{in}}\left(\mathbf{x}_{\mathrm{e}}, t_{\mathrm{e}}\right)=\frac{1}{c^{2}} \int_{\mathbf{x}_{\mathrm{s}} \in D_{\mathrm{s}}} \frac{\mathbf{n}_{\mathrm{s}} \times \partial_{t_{\mathrm{s}}} \mathbf{J}\left(\mathbf{x}_{\mathrm{s}}, t_{\mathrm{s}}\right)}{\left|\mathbf{x}_{\mathrm{s}}\right|} \mathrm{d} V_{\mathrm{s}},
\end{aligned}
$$

where $\mathbf{J}(\mathbf{x}, t)$ is the electric current density in the remote source. Time $t_{\mathrm{s}}$ in the right-hand-sides of (2)-(3) is retarded with respect to time $t_{\mathrm{e}}$.

The acceleration of a single electron due to these fields is given by the wellknown relativistic formula [7]

$$
\begin{aligned}
\partial_{t_{\mathrm{e}}} \mathbf{v}_{\mathrm{e}}=-\frac{e}{m_{\mathrm{e}}} \sqrt{1-\beta^{2}} & {\left[\mathbf{E}^{\mathrm{in}}\left(\mathbf{x}_{\mathrm{e}}, t_{\mathrm{e}}\right)-\beta^{2} \mathbf{n}_{\mathrm{v}}\left(\mathbf{n}_{\mathrm{v}} \cdot \mathbf{E}^{\mathrm{in}}\left(\mathbf{x}_{\mathrm{e}}, t_{\mathrm{e}}\right)\right)\right.} \\
& \left.+\beta \mathbf{n}_{\mathrm{v}} \times \mathbf{H}^{\mathrm{in}}\left(\mathbf{x}_{\mathrm{e}}, t_{\mathrm{e}}\right)\right]
\end{aligned}
$$

where $\beta=\left|\mathbf{v}_{\mathrm{e}}\right| / c, \mathbf{n}_{\mathrm{v}}=\mathbf{v}_{\mathrm{e}} /\left|\mathbf{v}_{\mathrm{e}}\right|$, and $\mathbf{v}_{\mathrm{e}}$ is the electron velocity. This formula clearly shows that we need to take into account the action of both the electric and the magnetic fields of the source. Subsequently, an electron radiates into the far-field the following electric field [7]:

$$
\mathbf{E}\left(\mathbf{x}_{\mathrm{o}}, t_{\mathrm{o}}\right)=-\frac{e}{c^{2}\left|\mathbf{x}_{\mathrm{o}}\right|\left[1-\beta\left(\mathbf{n}_{\mathrm{o}} \cdot \mathbf{n}_{\mathrm{v}}\right)\right]^{3}} \mathbf{n}_{\mathrm{o}} \times\left[\left(\mathbf{n}_{\mathrm{o}}-\beta \mathbf{n}_{\mathrm{v}}\right) \times \partial_{t_{\mathrm{e}}} \mathbf{v}_{\mathrm{e}}\right] .
$$




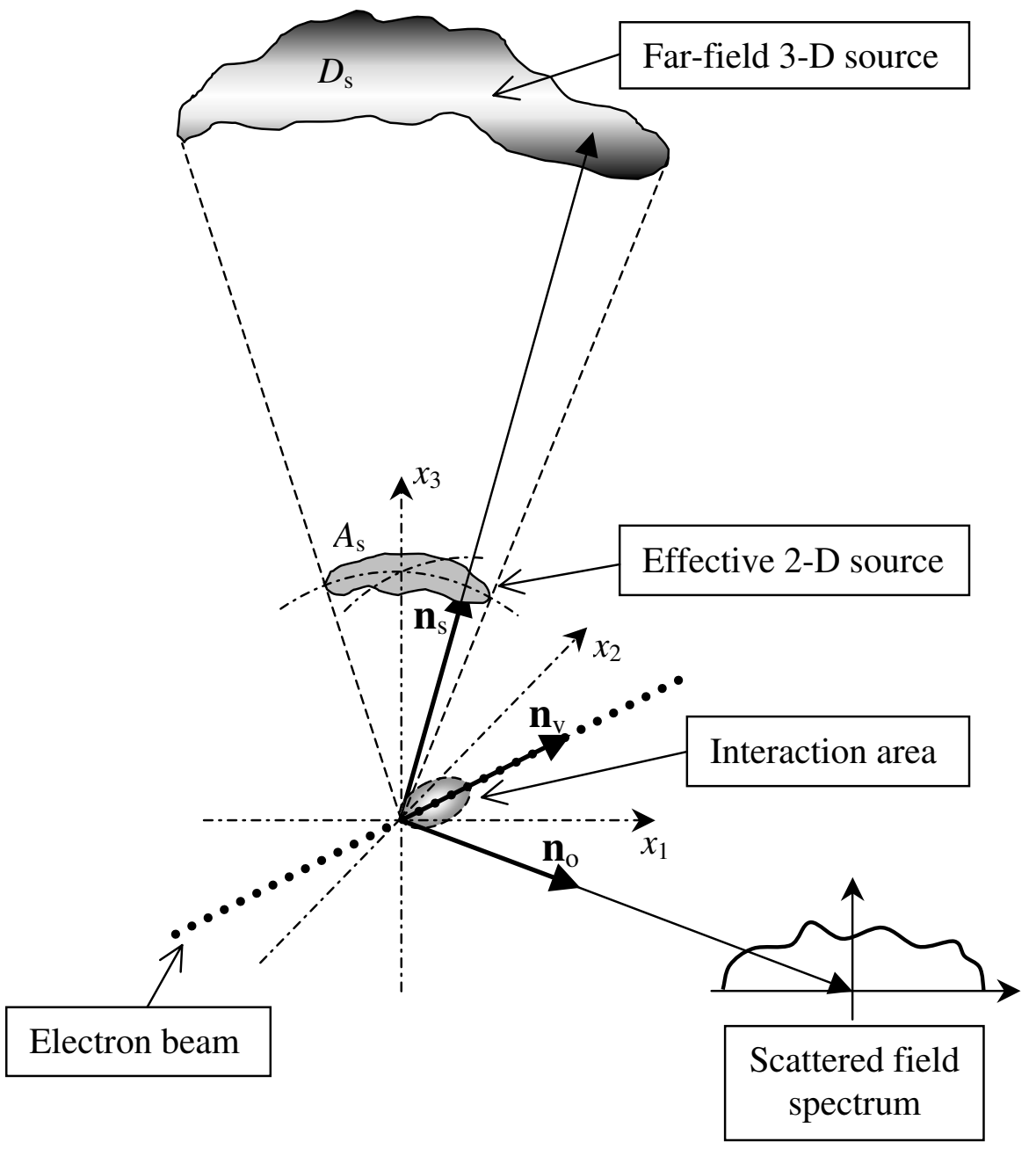

Fig. 1. Imaging setup with a relativistic detector (electron beam).

Here, $\mathbf{x}_{\mathrm{o}}$ denotes the location of the observation point. Time $t_{\mathrm{e}}$ in the righthand-side of this expression is retarded with respect to time $t_{\mathrm{o}}$. Upon substituting (2)-(3) in (4), and the result in (5), we arrive at

$$
\begin{aligned}
& \mathbf{E}\left(\mathbf{x}_{\mathrm{o}}, t_{\mathrm{o}}\right)= \frac{e^{2} \sqrt{1-\beta^{2}}}{c^{4} m_{\mathrm{e}}\left|\mathbf{x}_{\mathrm{o}}\right|\left[1-\beta\left(\mathbf{n}_{\mathrm{o}} \cdot \mathbf{n}_{\mathrm{v}}\right)\right]^{3}} \\
& \mathbf{n}_{\mathrm{o}} \times\left\{\left[\mathbf{n}_{\mathrm{o}}-\beta \mathbf{n}_{\mathrm{v}}\right] \times\left[\int_{\mathbf{x}_{\mathrm{s}} \in D_{\mathrm{s}}} \frac{\mathbf{n}_{\mathrm{s}} \times\left(\mathbf{n}_{\mathrm{s}} \times \partial_{t_{\mathrm{s}}} \mathbf{J}\left(\mathbf{x}_{\mathrm{s}}, t_{\mathrm{s}}\right)\right)}{\left|\mathbf{x}_{\mathrm{s}}\right|} \mathrm{d} V_{\mathrm{s}}-\right.\right. \\
& \beta^{2} \mathbf{n}_{\mathrm{v}}\left(\mathbf{n}_{\mathrm{v}} \cdot \int_{\mathbf{x}_{\mathrm{s}} \in D_{\mathrm{s}}} \frac{\mathbf{n}_{\mathrm{s}} \times\left(\mathbf{n}_{\mathrm{s}} \times \partial_{t_{\mathrm{s}}} \mathbf{J}\left(\mathbf{x}_{\mathrm{s}}, t_{\mathrm{s}}\right)\right)}{\left|\mathbf{x}_{\mathrm{s}}\right|} \mathrm{d} V_{\mathrm{s}}\right) \\
&\left.\left.+\beta \mathbf{n}_{\mathrm{v}} \times \int_{\mathbf{x}_{\mathrm{s}} \in D_{\mathrm{s}}} \frac{\mathbf{n}_{\mathrm{s}} \times \partial_{t_{\mathrm{s}}} \mathbf{J}\left(\mathbf{x}_{\mathrm{s}}, t_{\mathrm{s}}\right)}{\left|\mathbf{x}_{\mathrm{s}}\right|} \mathrm{d} V_{\mathrm{s}}\right]\right\}
\end{aligned}
$$


This expression describes the scattering by a single electron. The electric field scattered by a beam of non-interacting electrons is simply the sum of the fields scattered by each electron individually. All these fields can be computed using (6), provided that the electron velocities are given. In principle, these velocities are solutions of the relativistic equation of motion (4), which is a nonlinear equation and therefore very difficult, if not impossible, to solve. For our purposes, however, it is not necessary to know the velocities exactly. We rather need a reliable estimate on their variation with respect to the initial relativistic velocity.

To obtain such an estimate we use the Taylor expansion

$$
\mathbf{v}_{\mathrm{e}}\left(t_{0}+\Delta t\right)=\mathbf{v}_{\mathrm{e}}\left(t_{0}\right)+\left.\partial_{t} \mathbf{v}_{\mathrm{e}}\right|_{t=t_{0}} \Delta t+\text { higher order terms, }
$$

and observe that $\mathbf{v}_{\mathrm{e}}(t)$ can be approximated by the initial velocity up to the order $\Delta t$ - the time of the field-particle interaction. Note, if $\Delta t$ is sufficiently small, then the initial velocity approximation is suitable even for nonvanishing accelerations, and is, therefore, consistent with the idea of the secondary (i.e. acceleration related) radiation. Since $\Delta t$ is approximately the time of flight of electrons across the interaction area, one can decrease $\Delta t$ by decreasing this area. This can be achieved by either actually creating a relatively short relativistic current (radio frequencies) or by focusing the incident fields (optics), so that the latter are different from zero only within a certain finite and relatively small spatial domain. Under these assumtions we may take in (6)

$$
\begin{aligned}
\mathbf{v}_{\mathrm{e}}\left(t_{\mathrm{e}}\right) & \approx \mathbf{v}_{\mathrm{e}}\left(t_{0}\right)=\mathrm{constant} \\
\beta\left(t_{\mathrm{e}}\right) & \approx \beta\left(t_{0}\right)=\mathrm{constant} \\
\mathbf{n}_{\mathrm{v}}\left(t_{\mathrm{e}}\right) & \approx \mathbf{n}_{\mathrm{v}}\left(t_{0}\right)=\mathrm{constant}
\end{aligned}
$$

As far as the electron location $\mathbf{x}_{\mathrm{e}}\left(t_{\mathrm{e}}\right)$ is concerned, we are only interested in $\mathbf{x}_{\mathrm{e}}\left(t_{\mathrm{e}}\right)$ within the (small) area of interaction. For each electron this location depends on time linearly up to $\mathcal{O}(\Delta t)$. Under these assumptions the distance expression (1) becomes

$$
\left|\mathbf{x}_{\mathrm{s}}\right|-\mathbf{n}_{\mathrm{s}} \cdot \mathbf{x}_{\mathrm{e}}\left(t_{\mathrm{e}}\right) \approx\left|\mathbf{x}_{\mathrm{s}}\right|-t_{\mathrm{e}}\left(\mathbf{n}_{\mathrm{s}} \cdot \mathbf{v}_{\mathrm{e}}\right)+\mathbf{n}_{\mathrm{s}} \cdot \mathbf{x}_{\mathrm{e}}\left(t_{0}\right)
$$

where $\mathbf{x}_{\mathrm{e}}\left(t_{0}\right)$ is the initial location of the electron inside the area.

We set the origin of the coordinate system to be within the area of interaction. Recall that the times at the source and the electron locations are retarded with respect to each other and with respect to the time at the observation point. In the initial velocity approximation these times are

$$
\begin{aligned}
& t_{\mathrm{s}}=t_{\mathrm{e}}-\frac{\left|\mathbf{x}_{\mathrm{e}}\left(t_{\mathrm{e}}\right)-\mathbf{x}_{\mathrm{s}}\right|}{c} \approx t_{\mathrm{e}}\left[1+\beta\left(\mathbf{n}_{\mathrm{s}} \cdot \mathbf{n}_{\mathrm{v}}\right)\right]-\frac{\left|\mathbf{x}_{\mathrm{s}}\right|}{c} \\
& t_{\mathrm{e}}=t_{\mathrm{o}}-\frac{\left|\mathbf{x}_{\mathrm{o}}-\mathbf{x}_{\mathrm{e}}\left(t_{\mathrm{e}}\right)\right|}{c} \approx t_{\mathrm{o}}+t_{\mathrm{e}} \beta\left(\mathbf{n}_{\mathrm{o}} \cdot \mathbf{n}_{\mathrm{v}}\right)-\frac{\left|\mathbf{x}_{\mathrm{o}}\right|}{c}
\end{aligned}
$$


up to the order $\Delta t$. The arbitrary initial locations of electrons vanish, and, therefore, approximately the same time is now associated with all electrons inside the interaction area. From (10)-(11) we obtain the following relation between the times at the source location and the observation point:

$$
t_{\mathrm{s}}=\left(t_{\mathrm{o}}-\frac{\left|\mathbf{x}_{\mathrm{o}}\right|}{c}\right) \frac{1+\beta\left(\mathbf{n}_{\mathrm{s}} \cdot \mathbf{n}_{\mathrm{v}}\right)}{1-\beta\left(\mathbf{n}_{\mathrm{o}} \cdot \mathbf{n}_{\mathrm{v}}\right)}-\frac{\left|\mathbf{x}_{\mathrm{s}}\right|}{c} .
$$

A particularly simple expression in the case of almost constant velocities is obtained for the following choice of the observation direction:

$$
\begin{aligned}
\mathbf{n}_{\mathrm{o}} & =\mathbf{n}_{\mathrm{v}}, \\
\mathbf{n}_{\mathrm{o}} \cdot \mathbf{n}_{\mathrm{v}} & =1
\end{aligned}
$$

This choice reduces equation (6) to

$$
\begin{aligned}
\mathbf{E}\left(\mathbf{x}_{\mathrm{o}}, t_{\mathrm{o}}\right)= & \frac{N e^{2} \sqrt{1-\beta^{2}}}{c^{4} m_{\mathrm{e}}\left|\mathbf{x}_{\mathrm{o}}\right|(1-\beta)^{2}} \\
& \left\{\mathbf{n}_{\mathrm{v}} \times\left[\mathbf{n}_{\mathrm{v}} \times \int_{\mathbf{x}_{\mathrm{s}} \in D_{\mathrm{s}}} \frac{\mathbf{n}_{\mathrm{s}} \times\left(\mathbf{n}_{\mathrm{s}} \times \partial_{t_{\mathrm{s}}} \mathbf{J}\left(\mathbf{x}_{\mathrm{s}}, t_{\mathrm{s}}\right)\right)}{\left|\mathbf{x}_{\mathrm{s}}\right|} \mathrm{d} V_{\mathrm{s}}\right]\right. \\
& \left.+\beta \mathbf{n}_{\mathrm{v}} \times \int_{\mathbf{x}_{\mathrm{s}} \in D_{\mathrm{s}}} \frac{\mathbf{n}_{\mathrm{s}} \times \partial_{t_{\mathrm{s}}} \mathbf{J}\left(\mathbf{x}_{\mathrm{s}}, t_{\mathrm{s}}\right)}{\left|\mathbf{x}_{\mathrm{s}}\right|} \mathrm{d} V_{\mathrm{s}}\right\}
\end{aligned}
$$

where $N$ is the total number of electrons within the interaction area, which, presumably, is the same at any time instant.

Consider a single harmonic component of the time derivative of the source current density

$$
i \omega_{\mathrm{s}} \hat{\mathbf{J}}\left(\mathbf{x}_{\mathrm{s}}, \omega_{\mathrm{s}}\right) \exp \left(i \omega_{\mathrm{s}} t_{\mathrm{s}}\right)=i \omega_{\mathrm{s}} \hat{\mathbf{J}}\left(\mathbf{x}_{\mathrm{s}}, \omega_{\mathrm{s}}\right) \exp \left(-i \frac{\omega_{\mathrm{s}}}{c}\left|\mathbf{x}_{\mathrm{s}}\right|\right) \exp (i \omega t)
$$

where

$$
\begin{aligned}
& \omega=\omega_{\mathrm{s}} \frac{1+\beta\left(\mathbf{n}_{\mathrm{s}} \cdot \mathbf{n}_{\mathrm{v}}\right)}{1-\beta\left(\mathbf{n}_{\mathrm{o}} \cdot \mathbf{n}_{\mathrm{v}}\right)}=\omega_{\mathrm{s}} \frac{1+\beta\left(\mathbf{n}_{\mathrm{s}} \cdot \mathbf{n}_{\mathrm{v}}\right)}{1-\beta} \\
& t=t_{\mathrm{o}}-\frac{\left|\mathbf{x}_{\mathrm{o}}\right|}{c}
\end{aligned}
$$

Remarkably, frequency relation (16) coincides with the one obtained in [7] for the plane-wave case, which did not involve any approximations. We see now that even to a purely harmonic but spatially extended source there corresponds a whole set of frequencies in the scattered wave, which depend on the particular values admitted by $\mathbf{n}_{\mathrm{s}}$ and $\mathbf{n}_{\mathrm{v}}$ in (16). To obtain a more elaborate relation between the spatial distribution of the source current density and the spectrum 
of the scattered wave we take the Fourier transform of the observed electric field strength with respect to time. But first we simplify (14) by resorting to the effective two-dimensional aperture $A_{\mathrm{s}}$ (see Fig. 1), so that for a purely harmonic source (15) we have

$$
\begin{array}{r}
\mathbf{E}\left(\mathbf{n}_{\mathrm{v}}, t_{\mathrm{o}}\right)=C \int_{\mathbf{n}_{\mathrm{s}} \in A_{\mathrm{s}}}\left\{\mathbf{n}_{\mathrm{v}} \times\left[\mathbf{n}_{\mathrm{v}} \times \mathbf{e}\left(\mathbf{n}_{\mathrm{s}}, \omega_{\mathrm{s}}\right)\right]\right. \\
\left.+\beta\left[\mathbf{n}_{\mathrm{v}} \times \mathbf{h}\left(\mathbf{n}_{\mathrm{s}}, \omega_{\mathrm{s}}\right)\right]\right\} \exp (i \omega t) \mathrm{d} A,
\end{array}
$$

where $\mathbf{e}$ and $\mathbf{h}$ are the effective transverse fields with inhomogeneous angular distribution. All unimportant coefficients here and below are lumped with the constant of proportionality $C$. Notice that factor $\exp (i \omega t)$ cannot be taken outside the integral, since $\omega$ depends on the integration variable. The Fourier transform of the left-hand-side must be taken with respect to the observation time. Whereas, using the following simple manipulation:

$$
\begin{array}{r}
\hat{\mathbf{E}}\left(\mathbf{n}_{\mathrm{v}}, \omega_{\mathrm{o}}\right)=\int_{-\infty}^{\infty} \mathbf{E}\left(\mathbf{n}_{\mathrm{v}}, t_{\mathrm{o}}\right) \exp \left(-i \omega_{\mathrm{o}} t_{\mathrm{o}}\right) \mathrm{d} t_{\mathrm{o}}= \\
\exp \left(-i \frac{\omega_{\mathrm{o}}}{c}\left|\mathbf{x}_{\mathrm{o}}\right|\right) \int_{-\infty}^{\infty} \mathbf{E}\left(\mathbf{n}_{\mathrm{v}}, t+\frac{\left|\mathbf{x}_{\mathrm{o}}\right|}{c}\right) \exp \left(-i \omega_{\mathrm{o}} t\right) \mathrm{d} t
\end{array}
$$

in the right-hand-side we may take the Fourier transform with respect to retarded time $t$. The observed frequency is denoted $\omega_{\mathrm{o}}$. Now we substitute (18) in (19) and arrive at

$$
\begin{array}{r}
\hat{\mathbf{E}}\left(\mathbf{n}_{\mathrm{v}}, \omega_{\mathrm{o}}\right)=C \int_{\mathbf{n}_{\mathrm{s}} \in A_{\mathrm{s}}}\left\{\mathbf{n}_{\mathrm{v}} \times\left[\mathbf{n}_{\mathrm{v}} \times \mathbf{e}\left(\mathbf{n}_{\mathrm{s}}, \omega_{\mathrm{s}}\right)\right]\right. \\
\left.+\beta\left[\mathbf{n}_{\mathrm{v}} \times \mathbf{h}\left(\mathbf{n}_{\mathrm{s}}, \omega_{\mathrm{s}}\right)\right]\right\} \delta\left(\omega-\omega_{\mathrm{o}}\right) \mathrm{d} A,
\end{array}
$$

where $\delta\left(\omega-\omega_{\mathrm{o}}\right)$ is the Dirac delta-function, which for $\beta \neq 0$, and $\omega_{\mathrm{s}} \neq 0$, can be alternatively expressed as

$$
\begin{aligned}
\delta\left(\omega-\omega_{\mathrm{o}}\right) & =\delta\left[\omega_{\mathrm{s}} \frac{1+\beta\left(\mathbf{n}_{\mathrm{s}} \cdot \mathbf{n}_{\mathrm{v}}\right)}{1-\beta}-\omega_{\mathrm{o}}\right] \\
& =\frac{1-\beta}{\beta\left|\omega_{\mathrm{s}}\right|} \delta\left[\frac{\omega_{\mathrm{o}}(1-\beta)-\omega_{\mathrm{s}}}{\beta \omega_{\mathrm{s}}}-\left(\mathbf{n}_{\mathrm{s}} \cdot \mathbf{n}_{\mathrm{v}}\right)\right] .
\end{aligned}
$$

Upon substitution of this expression in (20), similarity with the Radon transform or, more precisely, the Doppler transform [2], [3] becomes obvious. The vectorial structure of equation (20) is still quite complex, however. The intensity of harmonics looks somewhat simpler:

$$
\begin{aligned}
(\overline{\hat{\mathbf{E}}} \cdot \hat{\mathbf{E}})= & \left(\overline{\mathbf{I}}_{\mathrm{e}} \cdot \mathbf{I}_{\mathrm{e}}\right)+\beta^{2}\left(\overline{\mathbf{I}}_{\mathrm{h}} \cdot \mathbf{I}_{\mathrm{h}}\right) \\
& -\left|\left(\mathbf{n}_{\mathrm{v}} \cdot \mathbf{I}_{\mathrm{e}}\right)\right|^{2}-\beta^{2}\left|\left(\mathbf{n}_{\mathrm{v}} \cdot \mathbf{I}_{\mathrm{h}}\right)\right|^{2}+2 \beta\left(\mathbf{n}_{\mathrm{v}} \cdot \operatorname{Re}\left\{\overline{\mathbf{I}}_{\mathrm{e}} \times \mathbf{I}_{\mathrm{h}}\right\}\right),
\end{aligned}
$$


where overbar denotes complex conjugation, and

$$
\begin{aligned}
& \mathbf{I}_{\mathrm{e}}=C \int_{\mathbf{n}_{\mathrm{s}} \in A_{\mathrm{s}}} \mathbf{e}\left(\mathbf{n}_{\mathrm{s}}, \omega_{\mathrm{s}}\right) \delta\left[\omega^{\prime}-\left(\mathbf{n}_{\mathrm{s}} \cdot \mathbf{n}_{\mathrm{v}}\right)\right] \mathrm{d} A, \\
& \mathbf{I}_{\mathrm{h}}=C \int_{\mathbf{n}_{\mathrm{s}} \in A_{\mathrm{s}}} \mathbf{h}\left(\mathbf{n}_{\mathrm{s}}, \omega_{\mathrm{s}}\right) \delta\left[\omega^{\prime}-\left(\mathbf{n}_{\mathrm{s}} \cdot \mathbf{n}_{\mathrm{v}}\right)\right] \mathrm{d} A .
\end{aligned}
$$

Splitting these up into components orthogonal and parallel to $\mathbf{n}_{\mathrm{v}}$, i.e.

$$
\mathbf{I}_{\mathrm{e}, \mathrm{h}}=\mathbf{I}_{\mathrm{e}, \mathrm{h}}^{\|}+\mathbf{I}_{\mathrm{e}, \mathrm{h}}^{\perp},
$$

we finally obtain

$$
(\overline{\hat{\mathbf{E}}} \cdot \hat{\mathbf{E}})=\left(\overline{\mathbf{I}_{\mathrm{e}}^{\perp}} \cdot \mathbf{I}_{\mathrm{e}}^{\perp}\right)+\beta^{2}\left(\overline{\mathbf{I}_{\mathrm{h}}^{\perp}} \cdot \mathbf{I}_{\mathrm{h}}^{\perp}\right)
$$

Subsequent transformations involve assumptions about the spatial coherence of the source, which are beyond the scope of this paper, but can be found for example in [9].

Anyway, it is clear that, if we consider two incoherent point sources, then the observed field has only two Fourier components corresponding to the frequencies, which can be easily determined from (16). In other words, we shall directly observe an image of our sources in the temporal spectrum of the field. Separation between the spectral lines depends on the angular separation of the sources and the electron velocity. The higher the velocity, the better the attainable spatial (angular) resolution.

\section{Discussion}

Unfortunately, there is no such thing in Nature as a purely monochromatic source of type (15). Therefore, even in the preceding simple one-dimensional example one should expect broadening of each of the two observed lines proportional to the broadening of the source's spectral line. In terms of the image quality this leads to the loss of spatial resolution - blurring. In addition, spectral resolution of the dispersing instrument (e.g. a prism or a grating) must be taken into account. Imagine two identical dispersing instruments. We place one of them on the path between the source and the interaction area, so that only one spectral line of width $\Delta \omega_{\mathrm{s}}$ is allowed through. Another dispersing instrument is used to investigate the spectral content of the scattered field. Then, for the two sources to be resolved, the electron velocity must satisfy

$$
\frac{\left|\mathbf{v}_{\mathrm{e}}\right|}{c} \geq \frac{1}{1+R \Delta \varphi}
$$


where $R=\omega_{\mathrm{o}} /\left|\Delta \omega_{\mathrm{o}}\right|$ is, by definition, the resolving power of a spectroscope, and $\Delta \varphi=\left|\sin \varphi_{1}-\sin \varphi_{2}\right|$ is the angular separation of the sources (the sources and the electron beam are coplanar).

Another anticipated cause of blurring is the variation of the particle velocity inside the interaction area, if the latter could not be made sufficiently small. The Fourier transform of (18) with a time-varying velocity will then introduce an extra convolution in $\omega_{\mathrm{o}}$-domain with the temporal Fourier transform of the electron velocity.

Correspondence between the spectrum and the source for two-dimensional distributions is not as direct as in the one-dimensional case. To invert the Radon transform spectral data for different directions of the velocity vector must be collected, i.e. we have to introduce the mutual rotation between the source and the particle accelerator around the axis perpendicular to the plane of the source. Subsequently, numerical inversion of the Radon transform must be carried out.

In summary, the proposed relativistic alternative to aperture-based imaging consists of employing a beam of relativistic electrons as a detector in an optical imaging system. A classical treatment of this problem has been presented. A more refined analysis would have to take into account: the mutual interaction between electrons, the radiation reaction force, and the quantum effects. The question of coherence must be investigated in more detail as well.

\section{Acknowledgement}

The author is grateful to Prof. A. T. de Hoop (Delft University of Technology) for his help and support. In particular, for his advice to tackle the problem using the relativistic equation of motion. The author also appreciates numerous discussions with Dr. R. Remis (Delft University of Technology).

\section{References}

[1] J. Bertrand and P. Bertrand, Microwave imaging of time-varying radar targets, Inverse Problems, Vol. 13 (1997), pp. 621-645.

[2] G. Sparr, K. Strählén, K. Lindström, and H. W. Persson, Doppler tomography for vector fields, Inverse Problems, Vol. 11 (1995), pp. 1051-1061.

[3] T. Schuster, The 3D Doppler transform: elementary properties and computation of reconstruction kernels, Inverse Problems, Vol. 16 (2000), pp. 701-722. 
[4] L. Desbat and C. Mennessier, On the invertibility of Doppler imaging: an approach based on generalized tomography, Inverse Problems, Vol. 15 (1999), pp. 193-213.

[5] Y. X. Huang, Identification of the property of a moving medium by the reflected electromagnetic wave, Radio Science, Vol. 38, No. 3 (2003), 1044.

[6] P. A. Fridman, On the possibility of aperture synthesis with a single mobile antenna in radio astronomy, IEEE Trans. Antennas and Propagation, Vol. 51, No. 7 (2003), pp. 1658-1662.

[7] L. D. Landau and E.M. Lifshitz, Classical Theory of Fields, Pergamon Press, London, 1962.

[8] F. Natterer and F. Wübbeling, Mathematical Methods in Image Reconstruction (SIAM Monographs on Mathematical Modeling and Computation), Philadelphia, PA: SIAM, 2001.

[9] M. Born and E. Wolf, Principles of Optics, Pergamon Press, London, 1959. 